\title{
COLORAÇÃO DO FRUTO, DISTÚRBIOS FISIOLÓGICOS E DOENÇAS EM MAÇÃS ‘GALA’ E 'FUJI’ PULVERIZADAS COM AMINOETOXIVINILGLICINA ${ }^{1}$
}

\author{
CASSANDRO VIDAL TALAMINI DO AMARANTE ${ }^{2}$ \\ CRISTIANO ANDRÉ STEFFENS ${ }^{3}$, LUIZ EDUARDO BASSAY BLUM ${ }^{4}$
}

RESUMO - O objetivo deste trabalho foi avaliar os efeitos da pulverização pré-colheita de macieiras 'Gala' e 'Fuji' com aminoetoxivinilglicina (AVG) sobre a qualidade dos frutos e a ocorrência de distúrbios fisiológicos e doenças. O experimento foi conduzido nos anos de 2005 e 2006, com pulverização de AVG (30 dias antes do início da colheita comercial dos frutos) nas doses de $0 ; 125$ e $250 \mathrm{mg} \mathrm{L}^{-1}$, sendo que, na segunda safra, também foi utilizada a dose de $62,5 \mathrm{mg} \mathrm{L}^{-1}$. Em maçãs 'Gala', o aumento na dose de AVG reduziu o desenvolvimento de coloração vermelha e a permeância à perda de água na casca. Nesta mesma cultivar, em frutos avaliados após armazenamento refrigerado (três meses a $0 \pm 0,5^{\circ} \mathrm{C} / 90-95 \% \mathrm{UR}$ ), seguido de uma semana de vida de prateleira $\left(20 \pm 4^{\circ} \mathrm{C} / 60-70 \%\right.$ UR), o tratamento pré-colheita com AVG aumentou a incidência de "bitter pit", porém reduziu a incidência de escaldadura, rachadura peduncular e podridões. Em maçãs 'Fuji', o aumento na dose de AVG reduziu a incidência e a severidade de pingo-de-mel em frutos colhidos tardiamente. A pulverização com AVG aumentou a severidade de mancha foliar de 'Gala' (Glomerella cingulata).

Termos de indexação: Malus domestica, "bitter pit", escaldadura, rachadura peduncular, pingo-de-mel, perda de água.

\section{FRUIT COLOR, PHYSIOLOGICAL DISORDERS AND DISEASES OF 'GALA' AND 'FUJI’ APPLES SPRAYED WITH AMINOETHOXYVINYLGLYCINE}

\begin{abstract}
The objective of this work was to study the effects of preharvest spraying of 'Gala' and 'Fuji' apple trees with aminoethoxyvinylglycine (AVG) in fruit quality and incidence of physiological disorders and diseases. The experiments were carried out in 2005 and 2006, with AVG sprayed (30 days before anticipated fruit harvest) at the doses of 0,125 and $250 \mathrm{mg} \mathrm{L}^{-1}$, and also at the dose of $62.5 \mathrm{mg} \mathrm{L}^{-1}$ in 2006. In 'Gala' apples, the increase of AVG dose delayed red color development and reduced the water loss permanence of the skin. For this cultivar, the preharvest treatment with AVG increased the incidence of bitter pit, and reduced the incidence of scald, stem-end splitting and decay on fruits assessed after cold storage (three months at $\left.0 \pm 0.5^{\circ} \mathrm{C} / 90-95 \% \mathrm{RH}\right)$, followed by one week of shelf life $\left(20 \pm 4^{\circ} \mathrm{C} / 60-70 \% \mathrm{RH}\right)$. The increase in AVG dose reduced the incidence and severity of watercore in late harvested 'Fuji' apples. The product increased the severity of 'Gala' leaf spot (Glomerella cingulata).

Index terms: Malus domestica, bitter pit, scald, stem-end splitting, watercore, water loss.
\end{abstract}

\section{INTRODUÇÃO}

A aminoetoxivinilglicina (AVG) inibe a conversão de S-adenosilmetionina (SAM) em ácido 1- aminociclopropano-1-carboxílico (ACC), catalisada pela enzima ACC sintase (ACS), na rota de síntese do etileno (Capitani et al., 2002). Devido a sua ação inibidora da síntese de etileno, a aplicação pré-colheita de AVG reduz a queda de frutos e retarda a maturação em maçãs 'Gala' (Amarante et al., 2002; Phan-Thien et al., 2004; Greene, 2005;
Argenta et al., 2006; Steffens et al., 2006; Petri et al., 2007), sendo estas respostas menos expressivas em maçãs 'Fuji', que apresentam menor produção de etileno (Amarante et al., 2002; Brackmann et al., 2004; Petri et al., 2007). A inibição da queda précolheita reduz as perdas, e o retardo na maturação permite flexibilidade na contratação de mão de obra para a colheita, classificação e empacotamento de frutos. Além disto, maçãs 'Gala' de plantas tratadas com AVG apresentam menores perdas de qualidade durante o armazenamento em atmosfera controlada

1(Trabalho 062-09). Recebido em: 09-03-2009. Aceito para publicação em : 19-10-2009.

${ }^{2} \mathrm{Ph}$.D., bolsista de produtividade em pesquisa do CNPq. Professor do Departamento de Agronomia, Centro de Ciências Agroveterinárias (CAV), Universidade do Estado de Santa Catarina (UDESC). Cx. Postal 281, CEP 88520-000, Lages- SC. Autor para correspondência. E-mail: amarante@cav.udesc.br

${ }^{3}$ Dr., Professor do Departamento de Agronomia, CAV/UDESC, Lages-SC. E-mail: steffens@cav.udesc.br

${ }^{4} \mathrm{Ph}$.D., bolsista de produtividade em pesquisa do CNPq. Professor do Departamento Fitopatologia, Instituto de Ciências Biológicas, Universidade de Brasília. Campus Universitário, Asa Norte, CEP 70910-900, Brasília-DF. E-mail: luizblum@unb.br 
(AC) (Brackmann \& Waclawovsky, 2001; Steffens et al., 2005; Drake et al., 2006).

A aplicação pré-colheita de AVG pode, no entanto, comprometer alguns aspectos qualitativos dos frutos. Em cultivares de macieiras que apresentam coloração vermelha da casca, o tratamento com AVG reduz a síntese de antocianinas na epiderme (Whale et al., 2008), além de retardar a degradação de clorofilas e o acúmulo de carotenoides (Huybrechts et al., 2003), comprometendo assim a qualidade visual dos frutos (Wang \& Dilley, 2001; Layne et al., 2002; Amarante et al., 2002; Schupp \& Greene, 2004; Byers et al., 2005; Greene, 2005; Argenta et al., 2006; Petri et al., 2007). O tratamento pré-colheita de macieira com AVG pode ainda reduzir a produção de voláteis aromáticos e a qualidade sensorial, avaliada nos frutos e no suco processado (Halder-Doll \& Bangerth, 1987; Drake et al., 2005a, 2005b e 2006). A pulverização pré-colheita das plantas com ethephon, após a aplicação de AVG, pode amenizar os efeitos negativos do AVG na redução da coloração vermelha (Wang \& Dilley, 2001; Steffens et al., 2006; Whale et al., 2008) e da qualidade sensorial (Drake et al., 2005a, 2005b e 2006).

A aplicação pré-colheita de AVG pode reduzir a incidência de doenças bióticas (Brackmann \& Waclawovsky, 2001; Steffens et al., 2005) e de distúrbios fisiológicos (doenças abióticas) pós-colheita em maçãs, tais como escaldadura (Ju \& Curry, 2000), pingo-de-mel (Brackmann et al., 2004; Drake et al., 2005a; Petri et al., 2006), rachadura peduncular e na casca (Andrews et al., 1999; Brackmann \& Waclawovsky, 2001; Steffens et al., 2005; Drake et al., 2006), polpa degenerescente e farinácea (Brackmann \& Waclawovsky, 2001; Steffens et al., 2005) e casca cerosa ('skin greasiness') (Lawes \& Woolley, 2001). Todavia, a aplicação pré-colheita de AVG aumentou a incidência de escurecimento interno da polpa em maçãs 'McIntosh' após oito meses de armazenamento em AC (Robinson et al., 2006). Em maçãs 'Cox's Orange Pippin', a aplicação pré-colheita de AVG ocasionou aumento substancial na incidência de degenerescência e escurecimento de polpa durante $o$ armazenamento em AC, com utilização de filtro catalítico para a remoção de etileno (Johnson \& Colgan, 2003). Portanto, a inibição da produção de etileno em maçãs tratadas com AVG pode reduzir a incidência de doenças bióticas e distúrbios fisiológicos relacionados com o amadurecimento e senescência. Porém, o etileno é importante na regulação do metabolismo celular, sendo que a inibição excessiva da sua síntese e/ou ação pode ocasionar a manifestação de certos distúrbios fisiológicos (Johnson \& Colgan, 2003). Em adição a isso, como o AVG é um inibidor de en- zimas que requerem piridoxal fosfato como ativador enzimático, além da enzima ACS, este composto pode inibir diversas outras enzimas envolvidas em processos metabólicos necessários para a regulação do amadurecimento dos frutos (Capitani et al., 2002). Como consequência, o AVG, dependendo da cultivar, da dose e da época de aplicação, pode ter efeito direto na manifestação de distúrbios fisiológicos durante o armazenamento refrigerado em maçãs. Mesmo em tecidos vegetativos de certas espécies de plantas, o tratamento com AVG pode ocasionar desordens fisiológicas. Em melões, a pulverização com AVG (260 $\mathrm{mg} \mathrm{L}^{-1}$ ), cerca de duas semanas antes da colheita, visando a retardar a maturação dos frutos, induziu fitotoxidez e clorose foliar, e redução no tamanho dos frutos (Shellie, 1999). Altas doses de AVG também inibiram o crescimento de maçãs 'Gala' (Amarante et al., 2002).

Este trabalho foi conduzido visando a estudar os efeitos da pulverização pré-colheita de macieiras 'Gala' e 'Fuji' com diferentes doses de AVG sobre a coloração dos frutos e a incidência de distúrbios fisiológicos e doenças.

\section{MATERIAL E MÉTODOS}

O experimento foi conduzido em pomar comercial localizado no município de Lages-SC, nos anos de 2005 e 2006. Macieiras com 11 a 12 anos de idade das cultivares Gala e Fuji, sobre porta-enxerto 'Marubakaido', foram pulverizadas cerca de quatro semanas antes do início da colheita dos frutos, com aminoetoxivinilglicina (AVG; ReTain ${ }^{\circledR}, 15 \%$ i.a. p/p, Sumitomo Chemical do Brasil Ltda.). No ano de 2005, foram utilizadas as doses de $0 ; 125$ e $250 \mathrm{mg}$ (i.a.) $\mathrm{L}^{-1}$, e no ano de 2006 , foi incluída a dose de 62,5 $\mathrm{mg} \mathrm{L}^{-1}$. Foi utilizado um pulverizador costal (20L), adicionando-se à calda (com $\mathrm{pH} \sim 7,0)$ o espalhante adesivo Silwet ${ }^{\circledR}(0,01 \% \mathrm{v} / \mathrm{v})$, fazendo-se a aplicação até o completo molhamento foliar.

Para ambas as cultivares, o experimento seguiu o delineamento em blocos ao acaso, com quatro repetições. Cada bloco consistiu em uma planta, e ramos laterais representaram as unidades experimentais. Cada dose de AVG foi aplicada em diferentes ramos da mesma planta, marcados aleatoriamente, eliminando-se assim qualquer variação imposta por diferenças quanto ao vigor entre plantas. Durante a aplicação, cada ramo recebendo pulverização do tratamento com AVG foi isolado dos demais com lona plástica.

Em macieiras 'Gala', as pulverizações com AVG foram efetuadas nos dias 14 e 15 de janeiro, nos anos de 2005 e 2006, respectivamente. No ano 
de 2005, os frutos foram colhidos nos dias 14 e 22 de fevereiro e 8 de março, correspondente a $0 ; 8$ e 22 dias após o início da colheita comercial, respectivamente. No ano de 2006, as colheitas foram realizadas nos dias 15 e 26 de fevereiro e 9 de março, correspondente a $0 ; 11$ e 22 dias após o início da colheita comercial, respectivamente. $\mathrm{Na}$ terceira data de colheita, foi omitido o tratamento-controle, pois apresentava um número reduzido de frutos devido à excessiva queda pré-colheita.

$\mathrm{Na}$ cultivar Fuji, os tratamentos foram efetuados nos dias 7 e 10 de março, nos anos de 2005 e 2006 , respectivamente. No ano de 2005 , as colheitas foram efetuadas nos dias 7 e 20 de abril e 4 de maio, correspondente a $0 ; 13$ e 27 dias após o início da colheita comercial, respectivamente. No ano de 2006 , os frutos foram colhidos nos dias 10 e 19 de abril e 3 de maio, correspondente a $0 ; 9$ e 23 dias após o início da colheita comercial, respectivamente.

Apenas em maçãs 'Gala', no ano de 2006, foi avaliado o efeito do AVG no desenvolvimento da coloração vermelha da epiderme dos frutos, a $0 ; 11$; 22 e 40 dias a partir do início da colheita comercial. A coloração foi avaliada em termos de superfície colorida (percentagem de cor vermelha), por meio de análise subjetiva visual, procedendo-se o cálculo da percentagem de frutos nas categorias 3 (Cat1; $<20 \%$ de superfície colorida), 2 (Cat2; 20-40\% de superfície colorida), 1 (Cat3; 40-60\% de superfície colorida) e extra ( $>60 \%$ de superfície colorida), segundo normas de classificação de maçãs do Brasil (2002). Mesmo frutos caídos no chão foram avaliados quanto à coloração, especialmente nas menores doses de AVG e nas últimas datas. Isto foi possível marcando todos os frutos de cada tratamento, nas plantas, com caneta de escrita permanente, permitindo assim a sua identificação quando caídos no chão.

Em cada data de colheita, maçãs 'Gala' e 'Fuji' (ambas com calibre 135; 127-141g) foram armazenadas em câmara fria convencional $\left(0 \pm 0,5^{\circ} \mathrm{C} / 90-95 \%\right.$ de umidade relativa) durante três e cinco meses, respectivamente, seguido de uma semana de exposição a condição ambiente $\left(20 \pm 4^{\circ} \mathrm{C} / 70-80 \% \mathrm{UR}\right)$. Os frutos de ambas as cultivares foram então avaliados quanto à incidência (\%) de "bitter pit", escaldadura e podridões e severidade de "bitter pit" (manchas/fruto). Apenas na cultivar Gala foi avaliada a incidência de rachadura peduncular interna (\%), já que é um distúrbio comum nesta cultivar (Andrews et al., 1999). Nesta cultivar, frutos da primeira colheita, no ano de 2005, foram avaliados quanto à permeância da casca à perda de água ( $P_{\mathrm{H}_{2 \mathrm{O}}}^{\prime} ; \mathrm{nmol} \mathrm{s}^{-1} \mathrm{~m}^{-2} \mathrm{~Pa}^{-1}$ ), segundo metodologia descrita $\mathrm{H}_{20} \mathrm{O}$ Amarante et al. (2001).

$\mathrm{Na}$ cultivar Gala, no ano de 2005, foi feita avaliação de severidade de mancha foliar (percentagem da área foliar com lesão e número de lesões por folha), ocasionada por Glomerella cingulata, dois meses após a pulverização com AVG (no dia 14 de março de 2005).

Apenas na cultivar Fuji, no ano de 2006, foi feita avaliação de incidência (\%) e severidade (nula $=0$; baixa $=1$; moderada $=2$, e alta $=3$ ) de pingode-mel nos frutos colhidos tardiamente, dois meses após a pulverização com AVG (no dia 10 de maio de 2006).

No ano de 2006, na data da primeira colheita, amostras de 20 frutos por repetição de cada tratamento, de ambas as cultivares, foram analisados quanto aos teores de $\mathrm{Ca}, \mathrm{K}, \mathrm{Mg}$ e $\mathrm{N}$ nos tecidos da casca e da polpa, segundo metodologia descrita por Amarante et al. (2006).

Os dados coletados foram submetidos à ANOVA, usando-se o programa SAS (Learning Edition, 2002). As médias de tratamentos (doses de AVG e data de colheita) foram comparadas pelo teste LSD, ao nível de 5\% de probabilidade. Dados de incidência e severidade de doenças e distúrbios fisiológicos foram também submetidos à análise de regressão para avaliar o efeito de dose de AVG.

\section{RESULTADOS E DISCUSSÃO}

Em todos os tratamentos, o atraso da colheita proporcionou redução no número de frutos Cat1, Cat2 e Cat3, e um aumento no número de frutos tipo Extra (Figura 1). Todavia, o aumento na dose de AVG diminuiu o percentual de frutos na categoria extra em todas as colheitas, notadamente em colheitas precoces. Frutos das plantas-controle, já na primeira colheita (dia zero), apresentavam $77,5 \%$ de frutos tipo Extra, enquanto plantas tratadas com AVG, nas doses de 62,5 e $125 \mathrm{mg} \mathrm{L}^{-1}$, somente atingiram esse valor em torno de 28 e 35 dias após o início da colheita comercial, respectivamente. Plantas tratadas com AVG, na dose de $250 \mathrm{mg} \mathrm{L}^{-1}$, não atingiram a percentagem de frutos tipo Extra da primeira colheita do tratamento-controle, mesmo 40 dias após o início da colheita comercial.

No Sul do Brasil, a pulverização de AVG na dose recomendada, de $125 \mathrm{mg} \mathrm{L}^{-1}$ (ou $125 \mathrm{~g}$ $\mathrm{ha}^{-1}$ ), cerca de um mês antes do início da colheita comercial, retarda a maturação de maçãs tipo 'Gala' em 8 a 18 dias (considerando a firmeza de polpa como indicador de colheita), dependendo da cultivar (Gala standard, Royal Gala ou Imperial Gala) e do ano agrícola (Amarante et al., 2002; Argenta et al., 2006; Steffens et al., 2006). Portanto, em maçãs 'Gala' tratadas com a dose recomendada de AVG 
(125 mg L $\left.\mathrm{m}^{-1}\right)$, mesmo colhendo os frutos 18 dias após a primeira data (dia zero), apenas $35 \%$ dos frutos seriam classificados como Extra, sendo que frutos Cat3, Cat2 e Cat1 corresponderiam a 7; 28 e 30\%, respectivamente (Figura 1).

Maçãs 'Fuji' são consideradas pouco suscetíveis ao "bitter pit" (Ferguson \& Watkins, 1989). Nesta cultivar, não houve ocorrência do distúrbio nos diferentes tratamentos avaliados, em ambos os anos (dados não apresentados). Em maçãs 'Gala', houve incidência de "bitter pit" em ambos os anos. No ano de 2006, a severidade do distúrbio foi bastante alto, superior a $25 \%$ em todos os tratamentos, não ocorrendo diferença entre doses de AVG (dados não apresentados). No ano de 2005, a incidência de "bitter pit" no tratamento-controle foi de $1-2 \%$, sendo que o tratamento com $\mathrm{AVG}$, nas doses de 125 e $250 \mathrm{mg} \mathrm{L}^{-1}$, aumentou a incidência e a severidade de "bitter pit" (avaliado após três meses de armazenamento refrigerado, seguido de uma semana de vida de prateleira), em frutos colhidos aos 0 e 8 dias após o início da colheita comercial (primeira e segunda colheitas) (Figuras 2A e 2B). Houve redução na intensidade de "bitter pit" nos frutos tratados com AVG com o retardo na data de colheita. Não houve diferença na severidade de "bitter pit" entre frutos colhidos aos 22 dias após o início da colheita comercial em plantas tratadas com AVG na dose de $125 \mathrm{mg} \mathrm{L}^{-1}$ e frutos do tratamentocontrole colhidos no dia zero (primeira data). No entanto, frutos da dose de $250 \mathrm{mg} \mathrm{L}^{-1}$, colhidos aos 22 dias após o início da colheita comercial, apresentaram maior severidade do distúrbio em relação ao controle colhido no dia zero.

O "bitter pit" é um distúrbio pós-colheita em maçãs, ocasionado pela deficiência de $\mathrm{Ca}$, e agravado pela existência de altos teores de $\mathrm{Mg}, \mathrm{K}$ e $\mathrm{N}$ nos frutos (Ferguson \& Watkins, 1989; Amarante et al., 2006). A análise nutricional dos frutos, em ambas as cultivares, não apresentou diferenças quanto aos teores de $\mathrm{Ca}, \mathrm{Mg}, \mathrm{K}$ e $\mathrm{N}$ nos tecidos da casca e da polpa entre tratamentos com AVG (dados não apresentados). Em maçãs 'Scarletspur Delicious', também não foi observado efeito do tratamento com AVG nos teores de minerais no suco dos frutos (Drake et al., 2005b). No entanto, além do aspecto nutricional, a incidência de "bitter pit" é maior em frutos colhidos em estádio menos avançado de maturação (Ferguson \& Watkins, 1989). Isto explica, em parte, a maior ocorrência de "bitter pit" em frutos tratados com AVG, em relação ao tratamento-controle, para uma mesma data de colheita (Figuras 2A e 2B), já que este composto retarda a maturação, pela inibição da síntese de etileno. Da mesma forma, a redução na ocorrência de "bitter pit" em frutos tratados com AVG, com o retardo na data de colheita, é em parte resultante do progresso da maturação dos frutos. Porém, frutos do tratamentocontrole, colhidos no dia zero, apresentaram menor severidade de "bitter pit" em relação aos frutos de plantas tratadas com AVG nas doses de 125 e $250 \mathrm{mg}$ $\mathrm{L}^{-1}$, e colhidos aos 8 e 22 dias após o início da colheita comercial, respectivamente. Para esses tratamentos e respectivas datas de colheita, não existem diferenças quanto ao estádio de maturação dos frutos (Amarante et al., 2002), mostrando que o AVG aumenta de fato a suscetibilidade de maçãs 'Gala' ao "bitter pit".

Maçãs 'Gala' são menos suscetíveis à escaldadura do que maçãs 'Fuji’ (Emongor et al., 1994). Todavia, no ano de 2005, houve grande incidência de escaldadura em 'Gala', não ocorrendo o distúrbio em 'Fuji'. No ano de 2006, não foi verificada ocorrência de escaldadura em ambas as cultivares. $O$ tratamento com AVG e o atraso na data de colheita reduziram a incidência de escaldadura em 'Gala' no ano de 2005 (Figura 2C). A suscetibilidade à escaldadura é maior em frutos colhidos antecipadamente (Emongor et al., 1994), o que parece explicar a redução na sua incidência com o retardo na colheita. Todavia, a causa provável do distúrbio é a biossíntese de $\propto$-farneseno no tecido da casca, e sua subsequente oxidação a trienos conjugados, ocasionando a degradação de células e o escurecimento do tecido (Emongor et al., 1994; Ju \& Curry, 2000). O etileno promove a biossíntese de $\propto$-farneseno e, portanto, aumenta a incidência de escaldadura (Emongor et al., 1994; Ju \& Bramlage, 2001). Portanto, a inibição da produção de etileno pela aplicação pré-colheita de AVG reduz a ocorrência de escaldadura em maçãs (Ju \& Curry, 2000; Ju \& Bramlage, 2001), conforme observado no presente trabalho.

A rachadura peduncular é um distúrbio fisiológico observado com frequência em maçãs 'Gala'. A antecipação da colheita e o aumento nas doses de AVG reduziram a incidência de rachadura peduncular em 'Gala' (Figura 2D). Através da inibição da síntese de etileno, o AVG retarda a degradação de parede celular, reduzindo assim a rachadura peduncular em 'Gala' (Andrews et al., 1999), da mesma forma como reduz a ocorrência de rachadura da casca dos frutos durante o armazenamento refrigerado (Steffens et al., 2005; Drake et al., 2006).

O AVG reduziu as podridões pós-colheita causadas por espécies de fungos fitopatogênicos, principalmente dos gêneros Alternaria, Botryosphaeria, Botrytis, Colletotrichum ou Penicillium, em maçãs 'Gala' armazenadas em câmara fria convencional durante três meses $\left(0-5^{\circ} \mathrm{C} / 90-95 \%\right.$ UR), seguido 
de uma semana de vida de prateleira $\left(20-22^{\circ} \mathrm{C} / 60\right.$ $70 \%$ UR), no ano de 2006. A incidência de doenças (valores médios de três datas de colheita) foi de $4,4 \%$ $( \pm 1,3) ; 3,8 \%( \pm 1,9) ; 2,0 \%( \pm 1,0)$, e $0,6 \%( \pm 0,4)$ nas doses de $0 ; 62,5 ; 125$ e $250 \mathrm{mg} \mathrm{L}^{-1}$ de AVG, respectivamente (modelo linear; $y=4,467-0,016 x$; $\left.\mathrm{R}^{2}=0,9643^{* * *}\right)$. Houve aumento na incidência de doenças pós-colheita com o atraso na colheita (dados não apresentados). Porém, não houve interação entre doses de AVG e datas de colheita. Estes resultados confirmam dados publicados por outros autores, mostrando redução de doenças pós-colheita em maçãs tratadas com AVG, devido ao retardo na maturação (Brackmann \& Waclawovsky, 2001; Steffens et al., 2005).

Em maçãs 'Fuji', não houve efeito de dose de AVG na incidência de doenças pós-colheita (dados não apresentados). Isto parece ser o resultado do pequeno efeito do AVG no retardo da maturação dos frutos nesta cultivar (Amarante et al., 2002; Brackmann et al., 2004; Petri et al., 2006). Em adição a isso, a cultivar Fuji apresenta alta suscetibilidade a doenças nos frutos, e é colhida tardiamente, sendo, portanto, submetida a um maior período de infecção por patógenos ainda no pomar. Em ambos os anos de execução do trabalho, foi observado $20-40 \%$ de incidência de doenças pós-colheita em maçãs 'Fuji'.

Na cultivar Fuji, o AVG reduziu a severidade e a incidência de pingo-de-mel em frutos colhidos dois meses após a pulverização do produto (Figura 3), possivelmente pela inibição da síntese de etileno (Wang \& Faust, 1992; Brackmann et al., 2004; Drake et al., 2005a; Petri et al., 2006). Todavia, mesmo com a utilização da dose de $250 \mathrm{mg} \mathrm{L}^{-1}$, correspondente ao dobro da dose recomendada para maçãs, houve apenas um controle moderado de pingo-de-mel. A incidência do distúrbio reduziu de $\sim 65 \%$ no tratamento-controle, para $\sim 45 \%$ na maior dose de AVG. Portanto, considerando este resultado e o alto custo de aplicação do produto, é questionável a viabilidade de sua aplicação para o controle de pingo-de-mel em 'Fuji'.

Em maçãs 'Gala', o tratamento com AVG reduziu a permeância da casca à perda de água, avaliada imediatamente após a colheita dos frutos no ano de 2005. Frutos dos tratamentos com 0; 125 e $250 \mathrm{mg} \mathrm{L}^{-1}$ de AVG apresentaram permeância de $31,8( \pm 1,3), 24,8( \pm 1,2)$ e $25,0( \pm 0,8) \mathrm{nmol} \mathrm{s}^{-1} \mathrm{~m}^{-2}$ $\mathrm{Pa}^{-1}$, respectivamente. A diferença foi altamente significativa $(p<0,001)$ entre o controle e as doses de 125 e $250 \mathrm{mg} \mathrm{L}^{-1}$ de $\mathrm{AVG}$, sendo que estas não diferiram entre si. Portanto, a pulverização com AVG nas doses de 125 e $250 \mathrm{mg} \mathrm{L}^{-1}$ reduz a permeância da casca de maçãs 'Gala' à água em 22\%, comparativamente ao controle, refletindo assim em menor perda de água dos frutos.

Segundo Ju e Bramlage (2001), o tratamento de maçãs 'Delicious' com AVG reduz o acúmulo de ácidos graxos livres totais e ceras totais na cutícula, pela sua ação inibitória da síntese de etileno. Lawes e Woolley (2001) também observaram redução na ocorrência de casca cerosa ('skin greasiness') em maçãs tratadas com AVG. Halder-Doll e Bangerth (1987) também reportaram um aspecto opaco e sem brilho da casca em maçãs 'Golden Delicious' tratadas em pré-colheita com AVG, ocasionada pela redução no conteúdo de ácidos graxos na cutícula. Todavia, segundo estes autores, o tratamento com AVG não afetou o conteúdo de ceras e aumentou o conteúdo de ácidos graxos saturados e monoinsaturados, em detrimento de ácidos graxos poli-insaturados na cutícula. Portanto, o aumento no grau de saturação de ácidos graxos na cutícula em frutos tratados com AVG parece explicar a redução na permeabilidade à perda de água (Amarante et al., 2001). Em adição a isso, maçãs 'Gala' tratadas com AVG apresentaram inibição no crescimento próximo da colheita (Amarante et al., 2002). O crescimento dos frutos próximo da colheita pode ocasionar a formação de imperfeições e rachaduras na cutícula, aumentando assim a sua permeância à perda de água (Amarante et al., 2001). Portanto, o menor crescimento em frutos tratados com AVG pode reduzir essas alterações na cutícula, reduzindo assim a perda de água.

O AVG aumentou a severidade de mancha foliar de 'Gala' (MFG), ocasionada por Glomerella cingulata, avaliada dois meses após a pulverização do produto, no ano de 2005 (Figura 4). Como o AVG é inibidor de enzimas que requerem piridoxal fosfato como ativador (Capitani et al., 2002), o produto pode comprometer a atividade de enzimas envolvidas em mecanismos de defesa dos tecidos à infecção de patógenos, aumentando assim a ocorrência de doenças, como observado para a MFG. Além disso, o etileno é importante na ativação de mecanismos de defesa dos tecidos vegetais à infecção por patógenos. A inibição da produção de etileno em plantas de Nicotiana benthamiana tratadas com AVG aumentou a queima foliar ocasionada por Botrytis cinerea (Chague et al., 2006). Baixas concentrações de etileno $\left(<1 \mu \mathrm{L} \mathrm{L}^{-1}\right)$, inferiores às normalmente produzidas, podem favorecer a germinação de esporos e a formação de apressório na fase anamórfica (Colletotrichum gloeosporioides) de G. cingulata, o que, por sua vez, pode facilitar a infecção (Prusky, 1996). Portanto, a inibição da produção de etileno nas folhas de macieiras 
'Gala' tratadas com AVG pode ter aumentado a suscetibilidade à MFG. $\mathrm{O}$ aumento na suscetibilidade à MFG pode ser ainda reflexo de efeito fitotóxico do produto nas folhas (Shellie, 1999).

Os resultados obtidos indicam que a pulverização pré-colheita com AVG, através da inibição da síntese de etileno, apresenta efeitos variados na qualidade e na ocorrência de doenças e distúrbios fisiológicos em maçãs, dependendo da cultivar e da dose utilizada. Em maçãs 'Gala' os principais efeitos negativos são o comprometimento na evolução de cor vermelha dos frutos, reduzindo assim frutos do tipo Extra, e o aumento na ocorrência de "bitter pit". Todavia, através da inibição da síntese de etileno, o produto reduziu a perda de água e a incidência de escaldadura, rachadura peduncular e podridões em 'Gala', e reduziu a incidência de pingo-de-mel em 'Fuji'. Todavia, em 'Gala', o produto aumentou a suscetibilidade das plantas a MFG.
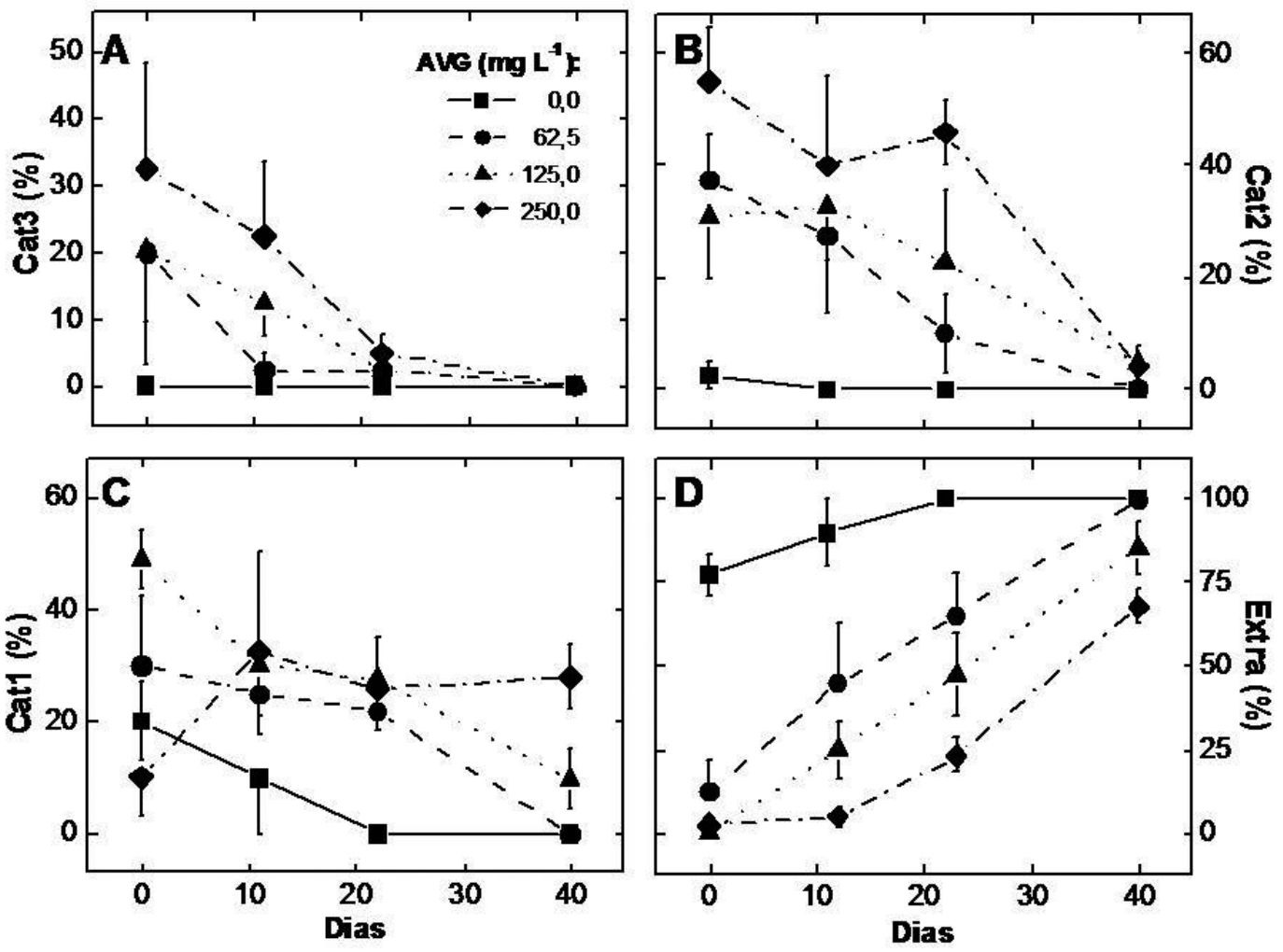

FIGURA 1 - Coloração vermelha de maçãs 'Gala', em função da dose de AVG e número de dias após o início da colheita comercial, segundo classificação em categorias 3 (Cat3; $<20 \%$ de superfície colorida) (A); 2 (Cat2; 20-40\% de superfície colorida) (B); 1 (Cat1; 40-60\% de superfície colorida) (C), e Extra ( $>60 \%$ de superfície colorida) (D). Valores médios $(n=4) \pm$ erro-padrão da média. Dados referentes ao ano de 2006. 

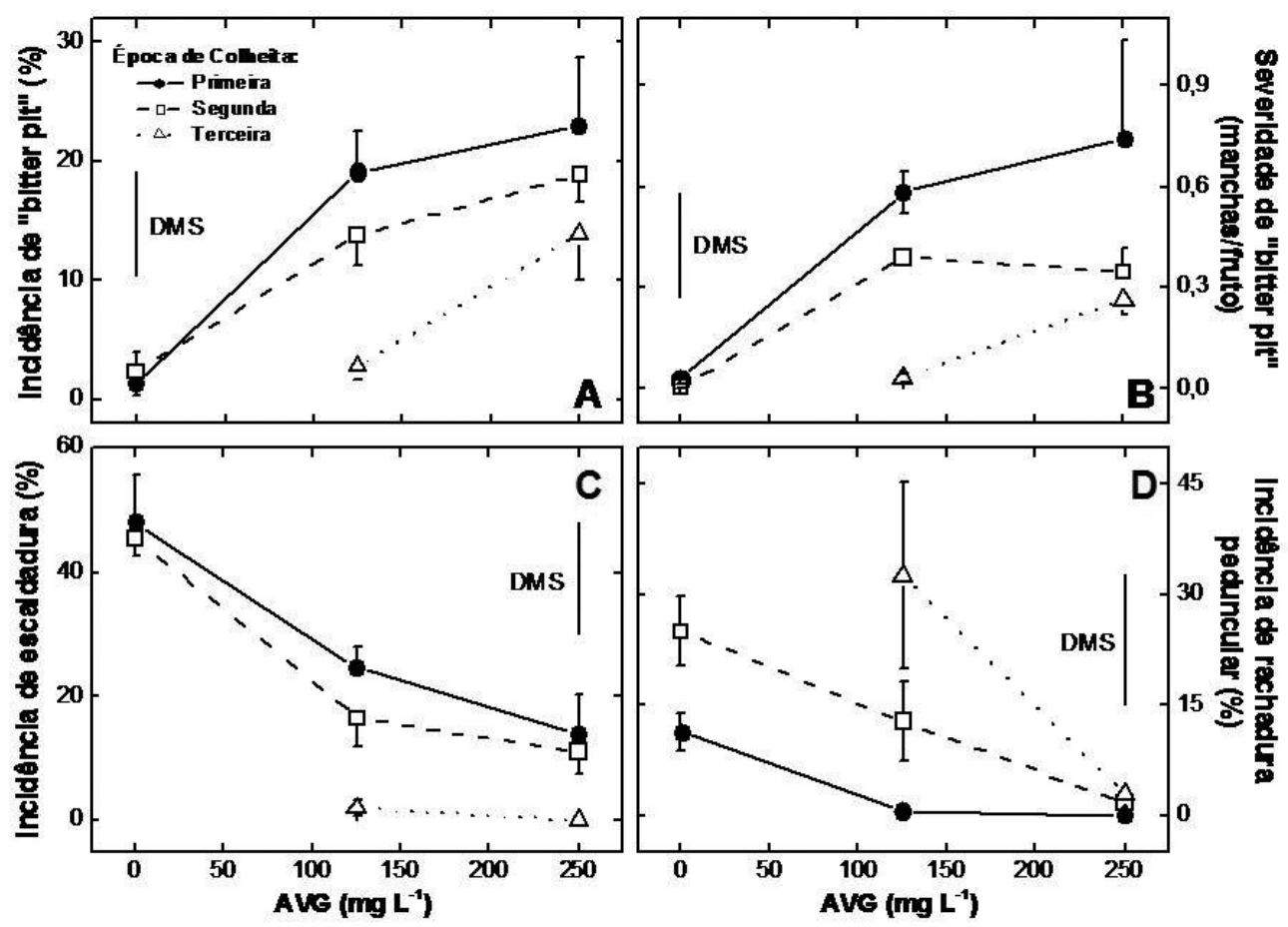

FIGURA 2 - Incidência (A) e severidade (B) de "bitter pit" e incidência de escaldadura (C) e rachadura peduncular interna (D) em maçãs 'Gala', pulverizadas na pré-colheita com diferentes doses de AVG, colhidas aos $0 ; 8$ e 22 dias após o início da colheita comercial (correspondente à primeira, segunda e terceira colheitas, respectivamente), e armazenadas em câmara fria convencional durante três meses $\left(0-5^{\circ} \mathrm{C} / 90-95 \%\right.$ UR), seguido de uma semana de vida de prateleira (20$22^{\circ} \mathrm{C} / 60-70 \%$ UR). Não são apresentados dados do tratamento-controle, aos 22 dias após o início da colheita comercial, devido à excessiva queda pré-colheita de frutos no mesmo. Barra vertical no interior da figura corresponde à diferença mínima significativa (DMS; $\mathrm{P}=0,05)$ entre valores médios ( \pm erro-padrão da média; $n=4$ ) das diferentes doses e datas de colheita. Dados referentes ao ano de 2005 .

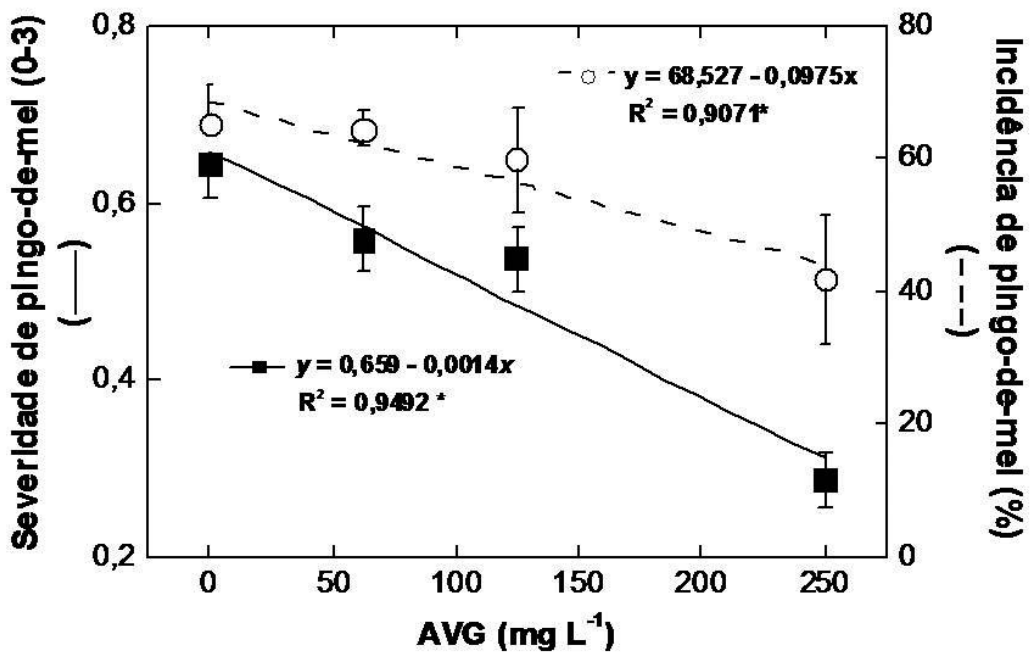

FIGURA 3-Severidade e incidência de pingo-de-mel em maçãs 'Fuji' pulverizadas na pré-colheita com diferentes doses de AVG, no dia 10-03-2006, e colhidas dois meses mais tarde (no dia 1005-2006). 


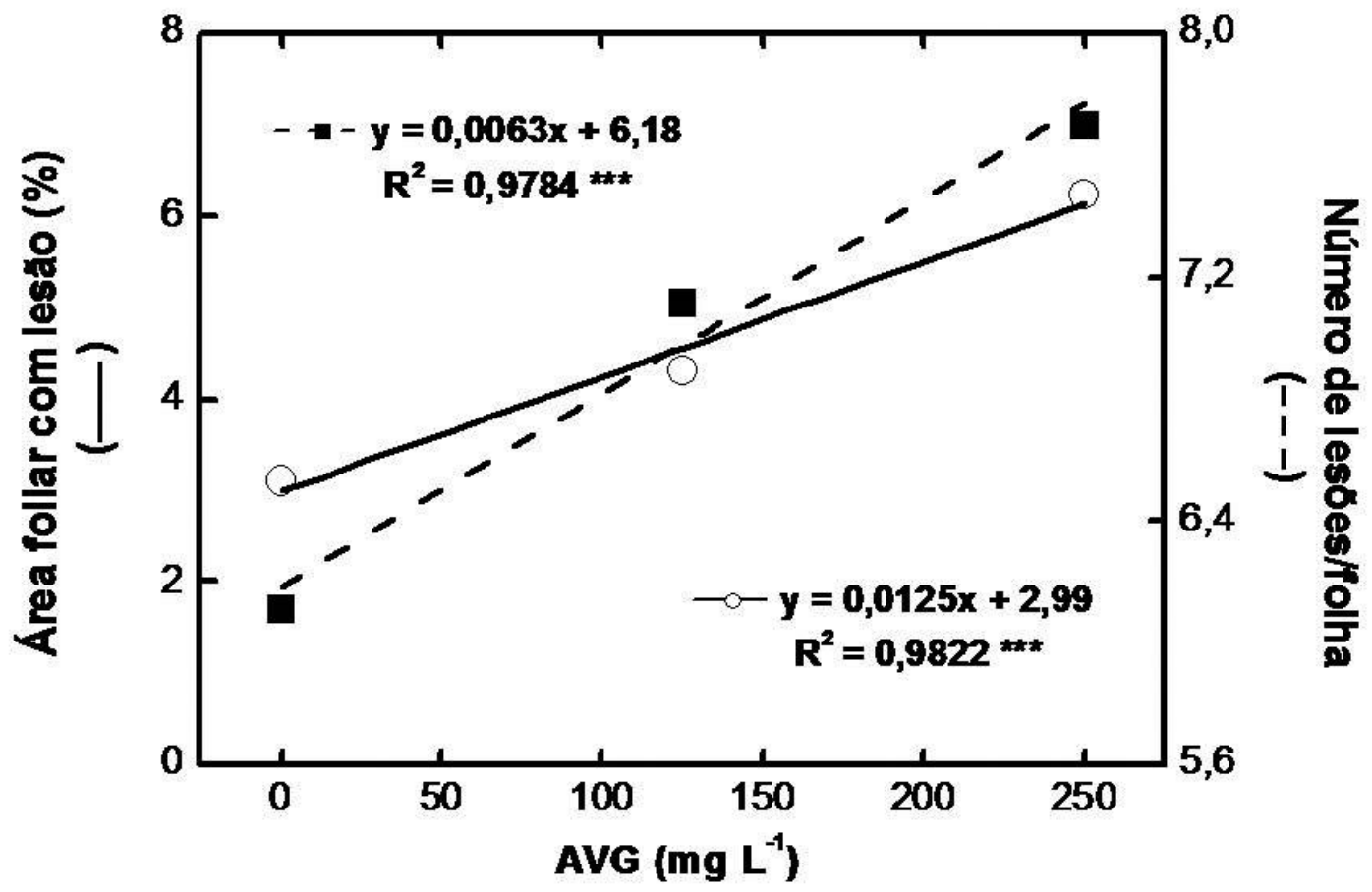

FIGURA 4 - Severidade (\% de área foliar afetada e número de lesões/folha) de mancha foliar (Glomerella cingulata) em macieiras 'Gala', pulverizadas na pré-colheita com diferentes doses de AVG, no ano de 2005. A pulverização com AVG foi efetuada no dia 13-01-2005, e a avaliação de doença foi feita no dia 14-03-2005.

\section{CONCLUSÕES}

1-Em maçãs 'Gala', a pulverização précolheita com AVG inibiu o desenvolvimento de coloração vermelha e reduziu a permeância à perda de água na casca dos frutos.

2-Maçãs 'Gala' tratadas com AVG apresentaram maior incidência de "bitter pit" e menor incidência de escaldadura, rachadura peduncular e podridões durante o armazenamento.

3-Em maçãs 'Fuji', a pulverização précolheita com AVG reduziu a intensidade de pingode-mel.

4-A pulverização pré-colheita com AVG aumentou a severidade de mancha foliar de 'Gala', ocasionada por Glomerella cingulata.

\section{AGRADECIMENTOS}

Ao Conselho Nacional de Desenvolvimento Científico e Tecnológico, por concessão de bolsas.

\section{REFERÊNCIAS}

ANDREWS, P.K.; COLLIER, M.L.; FAHY, D.; EVANS, R.G. Gala stem-end splitting and internal ring cracking. Good Fruit Grower, Yakima, v.50, n.11, p. 20-23, 1999.

AMARANTE, C.; BANKS, N.H.; GANESH, S. Relationship between character of skin cover of coated pears and permeance to water vapour and gases. Postharvest Biology and Technology, Amsterdam, v.21, n.3, p.291-301, 2001.

AMARANTE, C.V.T. do; SIMIONI, A.; MEGGUER, C.A.; BLUM, L.E.B. Effect of aminoethoxyvinylglycine $(A V G)$ on preharvest fruit drop and maturity of apples. Revista Brasileira de Fruticultura, Jaboticabal, v.24, n.3, p.661-664, 2002.

AMARANTE, C.V.T. do; CHAVES, D.V.; ERNANI, P.R. Análise multivariada de atributos nutricionais associados ao "bitter pit” em maçãs 'Gala'. Pesquisa Agropecuária Brasileira, Brasília, v.41, n.5, p.841846, 2006. 
ARGENTA, L.C.; VIEIRA, M.J.; KRAMMES, J.G.; PETRI, L.; BASSO, C. AVG and 1-MCP effects on maturity and quality of apple fruit at harvest and after storage. Acta Horticulturae, Leuven, n.727, p.495-503, 2006.

BRACKMANN, A.; WACLAWOVSKY, A.J. Responses of 'Gala' apples to preharvest treatment with AVG and low-ethylene CA storage. Acta Horticulturae, Leuven, n.553, v.1, p.155-157, 2001.

BRACKMANN, A.; STEFFENS, C.A.; GIEHL, R.F.H. Maturação da maçã 'Fuji' em função do atraso na colheita e da aplicação pré-colheita de aminoetoxivinilglicina. Ciência Rural, Santa Maria, v.34, n.3, p.737-742, 2004.

BRASIL. Ministério da Agricultura, Pecuária e Abastecimento. Instrução Normativa 50. Regulamento técnico de identidade e de qualidade para a classificação de maçã. 2002. 11p.

BYERS, R.E.; CARBAUGH, D.H.; COMBS, L.D. Ethylene inhibitors delay fruit drop, maturity, and increase fruit size of 'Arlet' apples. HortScience, Alexandria, v.40, n.7, p.2061-2065, 2005.

CAPITANI, G.; McCARTHY, D.L.; GUT, H.; GRÜTTER, M.G.; KIRSCH, J.F. Apple 1 -aminocyclopropane-1-carboxylate synthase in complex with the inhibitor L-aminoethoxyvinylglycine: evidence for a ketimine intermediate. The Journal of Biological Chemistry, Bethesda, v.277, n.51, p.49735-49742, 2002.

CHAGUE, V.; DANIT, L.V.; SIEWERS, V.; GRONOVER, C.S.;TUDZYNSKI, P.; TUDZYNSKI, B.; SHARON, A. Ethylene sensing and gene activation in Botrytis cinerea: a missing link in ethylene regulation of fungus-plant interactions? Molecular Plant Microbe Interactions, St. Paul, v.19, n.1, p.33-42, 2006.

DRAKE, S.R.; EISELE, T.A.; DRAKE, M.A.; ELFVING, D.C.; DRAKE, S.L.; VISSER, D.B. The influence of aminoethoxyvinylglycine and ethephon on objective and sensory quality of 'Delicious' apples and apple juice at harvest and after storage. HortScience, Alexandria, v.40, n.7, p.2102-2108, 2005a.
DRAKE, S.R.; EISELE, T.A.; ELFVING, D.C.; DRAKE, M.A.; DRAKE, S.L.; VISSER, D.B. Effects of the bioregulators aminoethoxyvinylglycine and ethephon on Brix, carbohydrate, acid, and mineral concentrations in 'Scarletspur Delicious' apple juice. HortScience, Alexandria, v.40, n.5, p.1421-1424, 2005b.

DRAKE, S.R.; ELFVING, D.C.; DRAKE, M.A.; EISELE, T.A.; DRAKE, S.L.; VISSER, D.B. Effects of aminoethoxyvinylglycine, ethephon, and 1-methylcyclopropene on apple fruit quality at harvest and after storage. HortTechnology, Alexandria, v.16, n.1, p.16-23, 2006.

EMONGOR, V.E.; MURR, D.P.; LOUGHEED, E.C. Preharvest factors that predispose apples to superficial scald. Postharvest Biology and Technology, Amsterdam, v.4, n.4, p.289-300, 1994.

FERGUSON, I.B.; WATKINS, C.B. Bitter-pit in apple fruit. Horticultural Reviews, New York, v.11, p.289-355, 1989.

GREENE, D.W. Time of aminoethoxyvinylglycine application influences preharvest drop and fruit quality of 'McIntosh' apples. HortScience, Alexandria, v.40, n.7, p.2056-2060, 2005.

HALDER-DOLL, H.; BANGERTH, F. Inhibition of autocatalytic $\mathrm{C}_{2} \mathrm{H}_{4}$-biosynthesis by $\mathrm{AVG}$ applications and consequences on the physiological behaviour and quality of apple fruits in cool storage. Scientia Horticulturae, Amsterdam, v.33, n.1/2, p.87-96, 1987.

HUYBRECHTS, C.J.G.; DECKERS, T.; VALCKE, R. Predicting fruit quality and maturity of apples by fluorescence imaging: effect of ethylene and AVG. Acta Horticulturae, Leuven, n.599, p.243-247, 2003.

JOHNSON, D.S.; COLGAN, R.J. Low ethylene controlled atmosphere induces adverse effects on the quality of Cox's Orange Pippin apples treated with aminoethoxyvinylglycine during fruit development. Postharvest Biology and Technology, Amsterdam, v.27, n.1, p.59-68, 2003.

JU, Z.; CURRY, E.A. Evidence that alpha-farnesene biosynthesis during fruit ripening is mediated by ethylene regulated gene expression in apples. Postharvest Biology and Technology, Amsterdam, v.19, n.1, p.9-16, 2000. 
JU, Z.; BRAMLAGE, W.J. Developmental changes of cuticular constituents and their association with ethylene during fruit ripening in 'Delicious' apples. Postharvest Biology and Technology, Amsterdam, v.21, n.3, p.257-263, 2001.

LAWES, G.S.; WOOLLEY, D.J. The commercial use of plant growth regulators to regulate fruit development. Acta Horticulturae, Leuven, n.553, v.1, p.149-150, 2001.

LAYNE, D.R.; WANG, J.-Z.; RUSHING, J.W. The influence of reflective film and ReTain on red skin coloration and maturity of 'Gala' apples. HortTechnology, Alexandria, v.12, n.4, p.640-645, 2002.

PETRI,J.L.; LEITE, G.B.; ARGENTA, L.C.; BASSO, C.; Ripening delay and fruit drop control in 'Imperial Gala' and 'Suprema' ('Fuji' sport) apples by applying AVG (Aminoethoxyvinylglycine). Acta Horticulturae, Leuven, n.727, p.519-524, 2006.

PETRI, J.L.; LEITE, G.B.; ARGENTA, L.C.; Eficácia do tratamento de AVG no controle da queda e maturação dos frutos de maçã, cultivar Imperial Gala. Revista Brasileira de Fruticultura, Jaboticabal, v.29, n.2, p.239-244, 2007.

PHAN-THIEN, K.Y.; WARGO, J.M.; MITCHELL, L.W.; COLLETT, M.G.; RATH,A.C. Delay in ripening of 'Gala' and 'Pink Lady' apples in commercial orchards following pre-harvest applications of aminoethoxyvinylglycine. Australian Journal of Experimental Agriculture, Collingwood, v.44, n.8, p.807-812, 2004.

PRUSKY, D. Pathogen quiescence in postharvest diseases. Annual Review of Phytopathology, Palo Alto, v.34, p.413-34, 1996.

ROBINSON, T.L.; WATKINS, C.B.; HOYING, S.A.; NOCK, J.F.; IUNGERMANN, K.I. Aminoethoxyvinylglycine and 1-methylcyclopropene effects on 'McIntosh' preharvest drop, fruit maturation and fruit quality after storage. Acta Horticulturae, Leuven, n.727, p.473-480, 2006.
SAS INSTITUTE. Getting started with the SAS learning edition. Cary: SAS, 2002. 200p.

SCHUPP, J.R.; GREENE, D.W. Effect of aminoethoxyvinylglycine (AVG) on preharvest drop, fruit quality, and maturation of 'McIntosh' apples. I. Concentration and timing of dilute applications on AVG. HortScience, Alexandria, v.39, n.5, p.10301035, 2004.

SHELLIE, K.C. Muskmelon (Cucumis melo L.) fruit ripening and postharvest quality after a preharvest spray of aminoethoxyvinylglycine. Postharvest Biology and Technology, Amsterdam, v.17, n.1, p.55-62, 1999.

STEFFENS, C.A.; GIEHL, R.F.H.; BRACKMANN, A. Maçã 'Gala' armazenada em atmosfera controlada e tratada com aminoetoxivinilglicina e ethephon. Pesquisa Agropecuária Brasileira, Brasília, v.40, n.9, p.837-843, 2005.

STEFFENS, C.A.; GUARIENTI, A.J.W.; STORCK, L.; BRACKMANN, A. Maturação da maçã 'Gala' com a aplicação pré-colheita de aminoetoxivinilglicina e ethephon. Ciência Rural, Santa Maria, v.36, n.2, p.434-440, 2006.

WANG, S.Y.; FAUST, M. Ethylene biosynthesis and polyamine accumulation in apples with watercore. Journal of the American Society for Horticultural Science, Alexandria, v.117, n.1, p.133-138, 1992.

WANG, Z.; DILLEY, D.R. Aminoethoxyvinylglycine, combined with ethephon, can enhance red color development without over-ripening apples. HortScience, Alexandria, v.36, n.2, p.328-331, 2001.

WHALE, S.K.; SINGH, Z.; BEHBOUDIAN, M.H.; JANES, J.; DHALIWAL, S.S. Fruit quality in 'Cripp's Pink' apple, especially colour, as affected by preharvest sprays of aminoethoxyvinylglycine and ethephon. Scientia Horticulturae, Amsterdam, v.115, n.4, p.342-351, 2008. 